\title{
Systems and Network Pharmacology Approaches to Cancer Stem Cells Research and Therapy
}

\author{
Irfana Muqbil', Ginny W Bao², Rkya El-Kharraj², Minjel Shah², Ramzi M Mohammad ${ }^{2,3}$, Fazlul H Sarkar ${ }^{2,4}$ and Asfar S Azmi*
}

${ }^{1}$ Department of Biochemistry, Aligarh Muslim University, Aligarh, Uttar Pradesh, India

${ }^{2}$ Department of Oncology, Barbara Ann Karmanos Cancer Institute, Detroit, Michigan, USA

${ }^{3}$ Hamad Medical Corporation, Doha, Qatar

${ }^{4}$ Department of Pathology, Wayne State University School of Medicine, Detroit MI 48201, USA

\begin{abstract}
The cancer stem cell (CSC) hypothesis is increasingly being accepted as a model to explain for the functional heterogeneity that is commonly observed in solid tumors. According to this hypothesis, there exists a hierarchical organization of cells within the tumor, in which a differential subpopulation of stem-like cells is responsible for sustaining and recurrence of tumor growth. CSCs have been shown to exist in a variety of solid tumors especially those with known resistant phenotypes such as breast, prostate and pancreatic adenocarcinoma (PDAC). In all these models, the commonality of deregulation of three crucial pathways; Wnt, notch and hedgehog that maintain CSC self-renewal capacity is emerging. Collectively these major pathways and have been linked to the observed resistance of CSC to chemotherapy and radiotherapy. The existing lack of knowledge and our incomplete understanding of the molecular signatures associated with CSCs highlight the need for better approaches in both isolation and identification of unique pathways associated with these cells. In this direction, computational biology, especially systems and network approaches, have proven to be of great utility in unraveling pathway complexities such as those associated with CSCs. With highlights on the most up-to-date molecular, network, cellular, clinical, and therapeutic cancer research findings, this article tends to provide a wealth of insights on systems and network biology approaches to CSC marker identification, the mechanism through which they evade treatment as well as therapeutic approaches that will help in conquering these elusive cells in incurable and refractory malignancies.
\end{abstract}

Keywords: Systems biology; Network pharmacology; Network medicine; Computational biology; Bioinformatics

\section{Introduction}

The concept that tumors arise from a rare population of cells with stem cell characteristics was first proposed more than a century ago when it was formulated that cancer results from the activation of embryonic-tissue. Since then, a number of advances in molecular biology and enhancement in diagnostic methods have led to their identification and characterization that has provided support to the cancer stem cell (CSC) hypothesis [1]. The first experiments indicating the existence of these cells were performed in animal models in the 1970s where it was concluded that only a low percentage of transplanted murine lymphoma cells formed colonies in the spleen of recipient animals [2]. This low in vivo and in vitro clonogenic potential of tumor cells was subsequently observed for cells isolated from human solid tumors and led to the proposal that the entire population of cells present in a certain tumor might be derived from a few cells which were defined as "tumor stem cells" [3]. Over the last few years the functional analysis of CSCs have been facilitated by advances in tissue culture, cell sorting, transgenic models and mouse-xenograft [4]. These advances have generated a considerable improvement in our knowledge of CSC's role in cancer and have made their selective targeting a focus of attention for successful cancer therapy. Nevertheless, the precise origin and functional properties of CSCs remains unclear or controversial [5]. Proponents of clonal selection theory claim that instead of stem cell theory, the hierarchical organization of a malignancy could be easily integrated in the classical clonal selection theory of Nowell [6]. This theory views a malignancy as a clonally-derived cell population, which acquires new potentially advantageous mutations that give rise to more rapidly proliferating clones. This leads to a process referred to as 'tumor Darwinism', which selects for the cell type most suitable for unlimited proliferation in the given environment. When one integrates the CSC theory in this model, the selection pressure is predicted to act at the level of the CSC compartment, implying that certain new traits in CSCs result in an increase in expansion of the CSCs due to self-renewal by symmetrical divisions.

Cancers contain a hierarchy of distinct populations of tumorigenic and non-tumorigenic cells. These cells might be more effectively studied and treated by focusing on the tumorigenic cells, particularly when those cells are rare. Therapies designed to eliminate only a small subpopulation of cancer cells will likely not have a clinical impact on cancers in which tumorigenic cells represent most of the cancer cells in the patient (Figure 1). Additional testing of the cancer stem cell model will be required in all cancers to determine what fraction of cases actually follow the model, and how often existing markers are informative. Such testing is likely to yield a complex picture involving differences between cancers, and even between patients with the same cancer, in terms of the frequency of tumorigenic cells, the degree of hierarchical organization, and the extent to which markers can distinguish tumorigenic from non-tumorigenic cells. The use of animal models is expected to lead to a better understanding of tumorigenesis in vivo and in the developmental relationship between cancer cells, and even new therapies. In this review, an attempt is made to extend some

*Corresponding author: Asfar S Azmi PhD, Department of Pathology, Wayne State University School of Medicine, Detroit, MI 48201, USA, Tel: 313-576-8327; Fax: 313-576-8389; E-mail: asfar_azmi@wayne.edu

Received November 16, 2012; Accepted December 14, 2012; Published December 16, 2012

Citation: Muqbil I, Bao GW, El-Kharraj R, Shah M, Mohammad RM, et al. (2012) Systems and Network Pharmacology Approaches to Cancer Stem Cells Research and Therapy. J Stem Cell Res Ther S7:005. doi:10.4172/2157-7633.S7-005

Copyright: ( 2012 Muqbil I, et al. This is an open-access article distributed under the terms of the Creative Commons Attribution License, which permits unrestricted use, distribution, and reproduction in any medium, provided the original author and source are credited. 
A

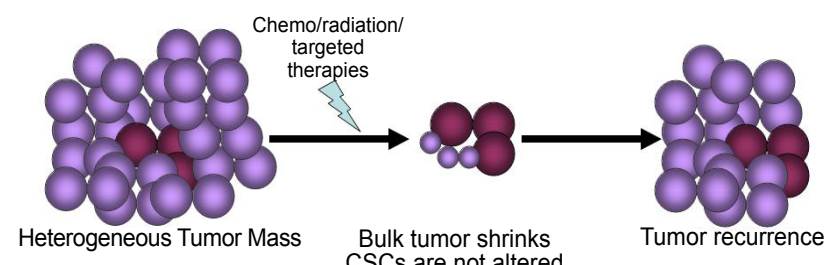

Cancer cells

Cancer stem cells (CSCs)

Figure 1: Cancer stem cells are resistant to conventional therapies. Tumors are heterogeneous and composed of both cancer cells (light purple) and a sub-population of highly resistant cell population that carry stemness markers or cancer stem cells (CSCs) (shown here in dark purple). Conventional chemotherapeutics, radiation and targeted therapies can eliminate the bulk of the tumors. However, the resistant sub-population of CSCs does not respond to most forms of therapies and potentially give rise to secondary tumors.

current ideas regarding the CSC hypothesis, through a systems and network perspective.

\section{Systems Understanding of Cancer Stems Cells}

Systems biology is a collective term encompassing different omics based disciplines that allow us to study the complex interactions within a biological system. The major difference separating traditional biology and systems sciences is that the latter relies on a more holistic perspective (holism instead of the reductionism) approach to biological and biomedical research. Since the beginning of this century, the concept has been used widely in the biosciences in a variety of contexts especially in cancer research [6]. One of the outreaching aims of systems biology is to model the underlying connectivity map of cells, tissues and organisms functioning as a system [7]. These emerging research approaches have proven of great utility in the study of cancer. In spite of the challenges [8], one area that has shown significant promise is in the mining of global gene expression data sets to identify molecular signatures that can be used for diagnosis and treatment selection [9]. As with any complex biological system, cancer (including cancer stem cells) can be viewed and interrogated at the genome-scale using systems biology approaches [10]. Systems biology has helped the understanding of biological information at previously unfathomable depths. For example the technology has allowed to simultaneously investigate the digital information of the genome and the influence of environmental information outside of it. Instead of honing on single gene or protein, through systems biology, researchers have been able to decipher in much greater detail the biological networks i.e. protein interactions and gene regulatory networks. Emerging systems tools are now being used to investigate gene regulatory networks, transcription factors and RNAs (lately non-coding RNAs or microRNAs as well) that control networks of other transcription factors and other RNAs. For example how information flows through signal-transduction networks, its integration and modulation that ultimately is conveyed and processed to networks of genes and proteins that execute developmental and physiological functions. The organization of these networks can be inferred from various different types of measurements including, for example, global measurements of dynamically changing levels of mRNAs and proteins during steady state and/or stress responses, as well as large-scale measurements of protein-protein and protein-
DNA interactions. Nevertheless, some drawbacks do exist such as in order to fully comprehend a biological system, information must be gathered from as many information levels as possible and integrated into models that generate testable hypotheses about how biological systems function (Figure 2A).

Following on the successes of molecular profiling in identifying prognostic signatures for many cancers, researchers have begun to perform profiling of cancer stem cells as well [11]. A number of studies have been done to find signatures for stemness in tumors. For example, gene signature for stemness in multiple cancer types has been identified

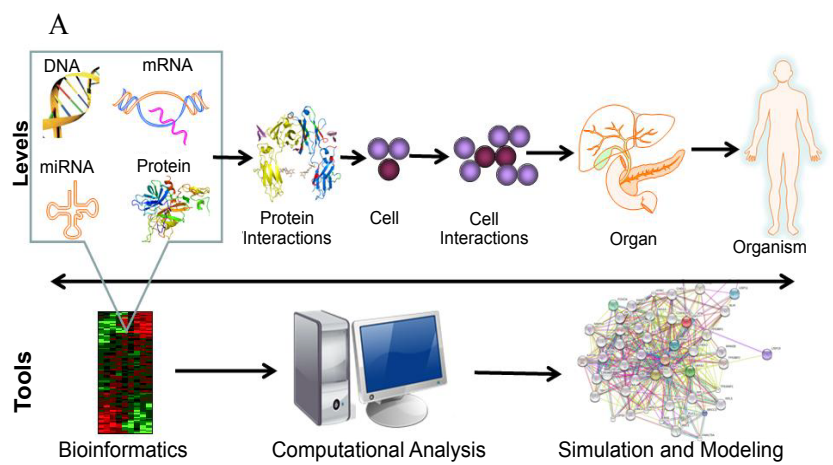

B $\mathrm{C}$

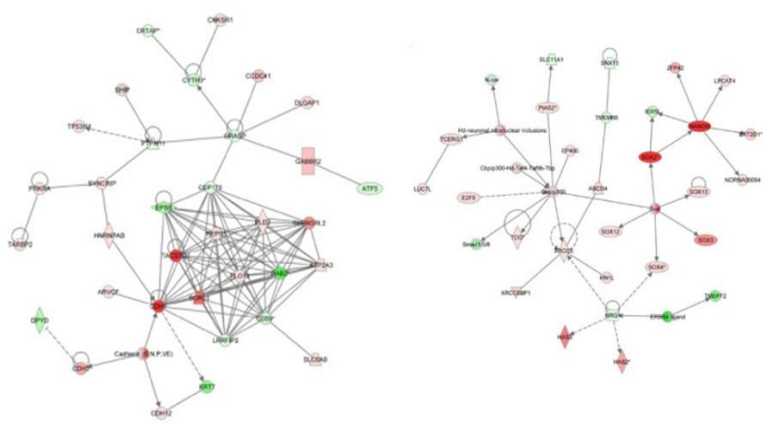

Figure 2: Systems biology. [A] Systems Biology is a conceptual framework for the analysis of complex biological systems from genomic information to the organismic level. Such systems derive from interactions among many distinct components (DNA, RNA, microRNA and proteins, in varying contexts (at the cellular, organ and organism level). These systems exhibit properties, such as nonlinear dynamics and emergent behavior that cannot easily be inferred from studies using reductionism. Systems biology relies on mathematical methods and computational models to generate hypotheses and to design new experiments that are driven from bioinformatics analysis. The quantity and quality of data required for these approaches often challenge current technologies, and development of new technologies, and cross-disciplinary collaborations, may be required. When applied to CSCs, systems biology can be a powerful tool to test hypotheses relevant to their development, role in cancer and provide avenues to novel therapeutic interventions. [B] Ingenuity analysis identifies novel gene networks involved in the maintenance of pluripotency. Genes over-expressed in non-human primate embryonic stem cells compared to fibroblasts are depicted in red. Genes shaded in green are over-expressed in fibroblasts. [C] Non-coding RNAs can be identified as members of pluripotent pathways using Ingenuity. Ingenuity analysis shows that genes known to be involved in stemness (Nanog and Sox-2) are overexpressed (red) in non-human primate embryonic stem cells (nhpESCs) compared to fibroblasts. In addition, we could identify non-coding RNAs that were over-expressed in nhpESCs and associated with Nanog. (figures 2B and 2C have been adopted from the Open Access article by Ahmi BenYehudah Stem Cell research and therapy). 
that were proven to predict important parameters such as interval to disease recurrence, metastasis and death $[12,13]$. The obtained signature highlighted B lymphoma Mo-MLV insertion region 1 homolog (BMl-1) oncogene-driven gene expression pathway, in which BMI-1 gene is essential for the self-renewal of hematopoietic and neural stem cells. Kaplan-Meier analysis, verified the utility of this stemness signature as predictive of survival in multiple tumor models. The above investigation showed that systems level evidence that the property of stemness is predictive of outcome in a wide variety of tumors. In another study, Turner and Kohandel presented both stochastic and deterministic models that explain tumor growth based on the CSC hypothesis and epithelial-mesenchymal transition [14,15]. In this study they demonstrated how quantitative computational approaches can be used to predict scenarios that can lead to an increase in CSCs following induction of epithelial-mesenchymal transition in human breast mammosphere models. These are some of the examples where systems sciences, particularly mathematical modeling has allowed for deeper evaluations of stemness markers in different tumor models.

\section{Molecular Networks of Cancer Stem Cells}

Systems approaches to CSCs involve not only the identification of the key components of a system through global analyses, but also information about how these components interacts in biological networks. Our understanding of biological network has benefited by the advancements in the concepts of network biology and network models of multiple types have been applied to a number of cancer systems. The most commonly applied models to cancer are interaction networks, including protein-protein interaction networks, protein-DNA and lately mRNA-microRNA interaction networks. Gene expression data can be used to identify differentially expressed genes subtype of tumor cells which can then be visualized on interaction networks. Various properties of these networks have been studied; with reported findings include the enrichment of CSC related genes among the "hubs" of the networks. While these interaction networks are very useful tools for differentiating large data sets, it is the predictive models stemming from mathematical descriptions of biochemical reaction networks and statistical influence models that are more useful in diagnosis and drug discovery. This is in light of the observation that gene/protein expression meta-analyses at the single gene/protein level have failed to identify a significant number of genes/proteins selectively expressed by a broad range of CSC types. Koeva and colleagues developed a computational method to test for CSC specific gene expression patterns from a comprehensive collection of 49 murine datasets covering 12 different stem cell types [16]. They hypothesized that stemness may be regulated by modules of homologs and although the expression of any single gene within a module may vary from one stem cell type to the next. It is possible that the expression of the module as a whole is required so that the expression of different, yet functionally-synonymous homologs is needed in different stem cells. In their comprehensive study they identified 40 individual genes and 224 stemness modules with reproducible and specific up-regulation across multiple stem cell types. The stemness modules included families regulating chromatin remodeling, DNA repair and Wnt signaling. Most intriguingly, their findings revealed that the majority of modules represent evolutionarily related homologs. Moreover, their score based on the discovered modules could accurately distinguish CSC populations from other cell types in both normal and cancer tissues. In another study, Zhong and colleagues constructed a general framework for modeling the genetic control of the dynamics of CSC populations that was based on the general characterization of a tumor [17]. Their model used specific differential equation parameters to describe different stages of tumor progression and test the magnitude and patterns of genetic effects on the proliferation of CSCs. The model provided clues to the differences in the proliferation dynamics of normal stem cells and CSCs. In principle this study allows the targeting of CSCs based on genetic data. The authors proposed that by coupling with "omics" data, their model may offer additional therapeutic window for success in specifically eliminating CSCs.

In this direction, Ben-Yehudah and colleagues using Ingenuity software identified system networks responsible for the regulation of the pluripotent state in nhpESCs and were able to create many gene networks (Figure 2B) [18]. The authors showed that most of these networks contained anticipated stem cell marker genes such as SOX2, OCT -4 and NANOG. Most interestingly, they were able to identify networks that have been shown to be differentially expressed between stem cells and other types of cells such as fibroblasts. The emergence of newer set of genes and their corresponding networks needs to be further explored for their relation to pluripotency. However, some genes such as TACSTD1 were found to play critical role in regulating the transition from pluripotency to differentiation. Most of the research has been carried out to understand the gene regulatory mechanisms that underlie regulation and control of pluripotency. These regulatory networks have been studied in mice and have shown the importance of key regulators of the stemness, including OCT-4, Sox-2 and Nanog. Using similar network methods, researchers have been able to construct microRNAs networks that control pluripotency (Figure 2C). It should be pointed out that although many genes and microRNAs have been implicated in the networks controlling pluripotency, little is known about the networks controlling this process. As the technology advances, we may get newer analysis tools that are capable of evaluating key regulators that control pluripotency.

From the above studies it is clear that that mathematical network modeling of gene regulatory networks in CSC may provide strong insights into the differences between normal stem cells and CSCs and some leads into new therapeutic formulations. Nevertheless superior network models that takes into account parameters such as feedback functions and therapeutic responses of cancer interventions are expected to drive future studies on CSCs.

\section{Network Approaches Identifying CSC Sweet Spot: Understanding CSC Metabolism}

Cancer metabolism has emerged as an important area of research in the last decade [19]. Elucidation of the metabolic differences between regular cancer cells and CSCs and the underlying mechanisms is expected to advance our understanding of fundamental CSC biology. Such advancements are projected to provide an important basis for the development of new therapeutic strategies and novel compounds to selectively eliminate CSCs by targeting their unique metabolism. Systems sciences have been helpful differentiating CSC metabolomic network from that of a regular cancer cells. This has benefitted by the recent stoichiometric reconstruction of known human metabolism at the genome level [20]. Researchers are subjecting such large scale metabolism reconstruction data to create initial models of the genomescale metabolic networks for CSCs. These studies may well provide insights into the unique metabolic features of cancer cells allowing one to identify both metabolic features that are shared or different among normal body cells, cancer cells and CSCs. However, the success of any metabolic network based strategy will depend on the improvements in CSC isolation procedures and their characterization.

Induced pluripotent stem cells (iPSCs) are a type of pluripotent 
stem cell artificially derived from a non-pluripotent cell by inducing a forced expression of specific genes [21]. These are extensively being studied as surrogate to CSC and have attracted great attention for possible application in drug screening and analysis of the mechanisms of cancer development, maintenance, progression and drug resistance. Utilizing network tools Shigeru Saito et al. analyzed both RNA profile to reveal gene expression changes and glycan profile to identify structural changes in glycans between parental somatic cell (SC) lines and their corresponding iPSCs [22]. Their combined statistical and network approach showed that there was a significant difference in expression patterns between the iPSCs and SCs. When subjected to network analysis, the gene expression and glycan signatures revealed a unique glycan transfer network associated with known epitopes for differentiation based on characteristic changes in the cellular surface states of the hiPSC. These studies showed that changes in exogenous networks affect iPSC metabolic network and have implications in developing drugs that work through re-programming of iPSC networks. Apart from the above studies, algorithms such as Reconstruction of Accurate Cellular Networks (ARACNe) are now also being applied to mammalian systems to obtain predictive networks for CSCs. Comparing CSC networks to those in normal stem cells and other tumor cells should prove highly informative in identifying drug targets unique to the CSC population of interest. Thus, computational modeling of CSC metabolic networks to identify potential therapeutic targets and to predict the effect of drug-induced perturbations is critical to this field moving forward.

\section{Cancer Stem Cell Network Targeted Drugs}

In most instances, chemotherapy, radiation and targeted therapies can eliminate bulk of the tumor cells. However, the highly resistant residual tumor cells especially CSCs are not affected by such treatments [23]. The residual CSCs can give rise to secondary tumor that ultimately results in tumor recurrence. Till date, most of the studies have focused on the design of single or combination regimens against tumor bulk. Although the non-specific and often time's highly toxic chemotherapeutics can successfully hit the cancer cell network, yet the success rate is limited and in most cases tumor rebounds in most of the solid tumors [24]. In order to have a successful anti-cancer therapeutic strategy, one needs to target both bulk tumor cells and CSCs (Figure 3). In an ideal scenario cancer can be cured by driving CSCs to extinction. Nevertheless, the problem of targeting CSCs is that ordinary stem cells will also be killed in the process. The dilemma is to design a therapeutic strategy that can selectively eliminate CSCs without harming normal hematopoietic stem cells. Here again computational and mathematical techniques have helped in designing strategies that target CSC specifically. Using a selective extinguishing strategy Sehl and colleagues delimited the killing differential i.e the difference between death rates of CSCs and normal stem cells [25]. Using Markov chains principle in continuous time they investigated the extinction times of CSCs vs that of normal stem cells. In their analysis system the application of extreme value theory from mathematical statistics yielded an accurate asymptotic distribution and corresponding moments for both extinction times. The authors were also able to identify additional parameters such as the impact of quiescence (the resting state) on CSC dynamics which was proposed to act as a salvage mechanism for CSCs thereby negating the effect of CSC targeted strategy. From these studies it was concluded that the proposed therapy must target quiescent CSCs as well as actively dividing CSCs. The above few mathematical studies indicate that successful targeting CSCs requires systems and network based approaches and some examples are discussed in the forthcoming passages.

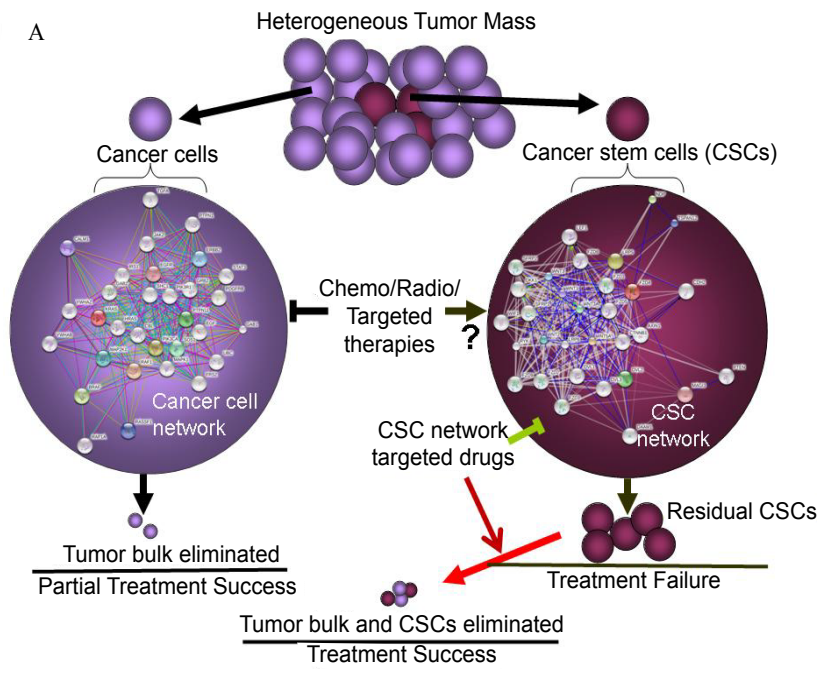

Figure 3: Cancer stem cell network targeted drugs for successful cancer therapy. Cancer cells and CSCs are sustained by unique protein-protein interaction (PPI) network. Chemo, radiation and other therapies can target cancer cell PPI leading to partial reduction of tumor (Left panel). On the other hand CSC networks are highly resistant and not affected by the standard regimens leading to recurring tumors (Right panel). For successful therapy a CSC network targeted regimen is needed and when combined with traditional chemotherapeutic may benefit the elimination of both cancer and CSCs.

\section{Promiscuous Drugs: The Future of CSC Therapies}

Complex cancers arise from alterations in multiple biological networks and carry heterogeneous cell lineages indicating that single pathway targeted therapy cannot eradicate the disease. To address this problem, clinicians routinely add multi-drug combinations with optimism that such additions will synergistically attack multiple pathways and different cell types within the tumor. Even though combination drug strategies that hit multiple target networks may increase efficacy, the consequent toxicity may limit the use of multidrug regimens. In this direction, very recently, drug discoverers have proposed that targeted drugs no matter how specific can hit multiple pathways leading to myriad secondary effects, some of them beneficial to the overall activity of the drug. This is especially true for CSCs that generally are resistant to most available chemotherapeutics and often times require very high doses to achieve any therapeutic response. So how can one target the resistant population of cells without increasing the toxicity? If one could weakly alter the molecular networks of CSCs to enhance sensitivity towards chemo and other forms of therapy then it is possible to eradicate these cells by lowering the doses of toxic drugs. The idea of re-programming resistant cell network to a sensitive one has spurred on another area of research that involves discovering agents with multi-targeted footprints i.e. having pleiotropic effects. Often times considered a cancer (here CSC) 'sweet spot' approach to drug discovery, it harnesses the overlap between pharmacologically relevant pathways, and the hits of chemical proteomics, which represent those proteins, as these can potentially interact with druggable molecules [26]. In cancer/CSCs, the 'sweet spot' represents those few hundred proteins, which are both parts of disease relevant pathways and are druggable or re-programmable. On one hand, the option to allow indirect effects via network-contacts of multi-target drugs expands the target horizon, since the number of those proteins, which are indirectly related to existing targets of pharmacologically important pathways, is by several magnitudes greater than the number of the targets themselves. On the other hand, the low-affinity binding of multi-target 
drugs eases the constraints of druggability. Fitting this tall order, the pleiotropic natural plant-derived agents show promise against cancer specially CSC [27-29]. Natural agents are not new to cancer research and as a matter of fact more than $40 \%$ of all the existing cancer drugs have been derived from natural agents and/or have nature's footprints [30]. Leading this flagship is Taxol that is successfully used for various cancers. Taxol was discovered at the US National Cancer Institute (NCI) in the late 1960s in the course of an in vitro antitumor drug discovery program using human tumor cell lines. Screening of more than 110,000 samples derived from more than 35,000 plant genera collected worldwide resulted in the isolation and structure elucidation of Taxol from the bark of the Californian yew tree Taxus brevifolia [31]. In general, most these agents are non-toxic and many have been a part of the human diet since ancient times. These agents have been extensively studied and have been shown to weakly modulate cancer cell signaling thereby resulting in cancer cell selective apoptosis. Based on these important properties, we propose these highly promising agents as excellent candidates in promiscuous strategies against CSCs either alone or in combination with standard chemotherapy $[32,33]$. Some of these agents have been investigated for their activity against CSCs using traditional molecular biology and network sciences and are discussed below.

\section{Utilizing Network Pharmacology to Harness the Potential of Pleiotropic Natural Agents against CSCs}

As mentioned previously, natural agents (especially plant derived) have been extensively investigated for their cancer preventive and therapeutic benefits [34-36]. Over the last three decades researchers have tried to identify the exact mechanism behind their anti-cancer activities and as well as their cancer cell selectivity. Recently our laboratory has investigated their potential against prostate and pancreas CSCs. As identified by network analysis, the vast majority of the CSC cellular protein, signaling and transcriptional networks are in a low-affinity or transient weak linkage with each other i.e. forming a complex network. Therefore, natural agents serve as excellent model systems where network pharmacology can be applied to predict their scope of action against a particular cancer (Figure 4 summarizes how complex networks can be targeted by promiscuous drugs).

CDF (curcumin difluorinated) is a difluoro analog of the wellrecognized chemopreventive agent curcumin [37]. It is a multi-targeted agent with proven anti-cancer effects in vitro and in vivo against CSCs [38-42]. Similar to multi-targeted agents such as multi-kinase inhibitors etc, CDF was found to have lower target binding affinity than a single-target drug on multiple macromolecules. For example, in one study using network modeling, we demonstrated that CDF modulates a number of microRNAs and that in turn regulate c-Myc hub and these perturbations orchestrate a selective set of events that eventually led to the induction of apoptosis in pancreatic cancer cells [43]. Befitting the multi-targeted agent model, the same drug has been reported to influence the pathways directly supporting CSCs such as epithelial mesenchymal, NF-kB signaling and stemness miRNAs as well. It is highly possible that there may be other potential targets of CDF that are context driven and tumor dependent and still yet to be discovered. Another agent Diindolylmethane (BR-DIM), has been extensively investigated by our laboratory for its CSC suppressive effects. BRDIM primarily inhibits CSC by targeting the NF-kB signaling. Nevertheless, numerous other mechanisms have also been proposed that include enhancement of pro-apoptotic proteins such as prostate apoptosis response 4 (Par-4) [44], MMP9 [45], uPA [46,47], FOXO3 [48] and inhibition of the mTOR pathway [49]. Most importantly

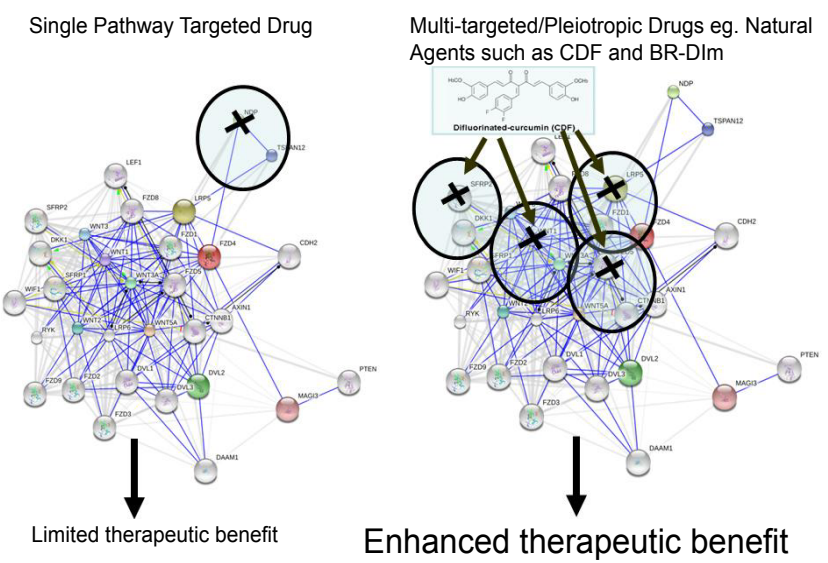

Figure 4: Multi-target drugs have a broader impact on complex network. (Left panel) Single pathway targeted drugs have limited scope in complex networks (such as those related to CSCs). A very specific drug restricts its activity to a single protein that may or may not be part of bottleneck module. (Right panel) On the other hand, multi-targeted natural agents such as CDF have been recognized to myriad targets. For example transcription factors such as NF-kB, myc and notch that are established components of bottleneck modules. Therefore, such pleiotropic agents act as target multipliers and increase the target radius thereby showing superior cancer inhibitory effects.

and in suport of our hypothesis both CDF and BR-DIM act as potent chemosensitizers and possibly work through re-programming of the CSC molecular network. In order to explore this hypothesis, our group recently utilized network analysis to identify the set of target genes of this multi-faceted agent. By pathway and network analysis, we observed the regulatory effects of isoflavone and BR-DIM on multiple signaling networks such as AR/PSA, NKX3-1/Akt/p27, MITF, etc [50-52]. These studies suggest that BR-DIM with their multi-targeted effects could be useful for the prevention of progression, particularly by attenuating bone metastasis mechanisms. Our systems level investigations have ushered BR-DIM into the clinic for patients with Stage I or stage II prostate cancer undergoing radical prostatectomy. In essence, our proof of concept and translational investigations demonstrate that systems and network based studies could be useful in designing promiscuous strategies against CSCs. These novel strategies would likely aid in the future designing and development of successful therapeutic regimens for cancers carrying heterogeneous mass of cells.

\section{Conclusion}

The identification and prospective isolation of CSCs from both solid tumors and hematological malignancies has spawned a new paradigm in cancer research. From the perspective of systems biology it is envisioned that increasingly global assessment of CSCs and their microenvironments (niche) at the level of complete transcriptome, proteome, and epigenome, using empowering new high throughput technologies will allow researchers to design successful therapeutic strategies not only against the bulk of the tumor (comprising of cancer cells) but also against these highly resistant CSCs. Emerging systems technologies are expected to allow researcher for superior identification of better cell-surface markers and their interaction with the resident CSC niche and potential diagnostic markers from both body fluids and tumor tissues. Additionally, the protein-protein interaction networks of CSCs are expected to serve as more accurate indicatives for diagnosis and prognosis. Incorporating these data into biological networks will provide fundamental insights into the biology of cancer stem cells and 
Citation: Muqbil I, Bao GW, El-Kharraj R, Shah M, Mohammad RM, et al. (2012) Systems and Network Pharmacology Approaches to Cancer Stem Cells Research and Therapy. J Stem Cell Res Ther S7:005. doi:10.4172/2157-7633.S7-005

their abilities for renewal and differentiation. The complexity of the interaction networks in CSCs indicate that promiscuous strategies such as multi-targeted natural agents and not the single pathway approach is the way forward for success against these elusive cells. These combined efforts will ultimately lead to new therapeutic strategy specifically targeting cancer stem cells for unprecedented personalized cancer therapy.

\section{Acknowledgment}

National Cancer Institute, NIH grants R01CA109389 and R21CA169848 to R.M. Mohammad are acknowledged.

\section{References}

1. Gilbertson RJ, Graham TA (2012) Cancer: Resolving the stem-cell debate. Nature [Epub ahead of print]

2. Park $\mathrm{CH}$, Bergsagel DE, McCulloch EA (1971) Mouse myeloma tumor stem cells: a primary cell culture assay. J Natl Cancer Inst 46: 411-422.

3. Hamburger AW, Salmon SE (1977) Primary bioassay of human tumor stem cells. Science 197: 461-463.

4. Qin J, Liu X, Laffin B, Chen X, Choy G, et al. (2012) The PSA(-/lo) prostate cancer cell population harbors self-renewing long-term tumor-propagating cells that resist castration. Cell Stem Cell 10: 556-569.

5. Jordan CT (2009) Cancer stem cells: controversial or just misunderstood? Cell Stem Cell 4: 203-205.

6. Nowell PC (2002) Tumor progression: a brief historical perspective. Semin Cancer Biol 12: 261-266.

7. Hood L, Tian Q (2012) Systems approaches to biology and disease enable translational systems medicine. Genomics Proteomics Bioinformatics 10: 181185

8. Brennan MD, Cheong R, Levchenko A (2012) Systems biology. How information theory handles cell signaling and uncertainty. Science 338: 334-335.

9. Zhao Z, Jiang R, Zheng H (2012) Systems biology approaches in biological and biomedical research: opportunities and challenges. Int J Comput Biol Drug Des 5: 181-184.

10. Wang IM, Stone DJ, Nickle D, Loboda A, Puig O, et al. (2012) Systems Biology Approach for New Target and Biomarker Identification. Curr Top Microbiol Immunol [Epub ahead of print].

11. Price ND, Foltz G, Madan A, Hood L, Tian Q (2008) Systems biology and cancer stem cells. J Cell Mol Med 12: 97-110.

12. Ho Sui SJ, Begley K, Reilly D, Chapman B, McGovern R, et al. (2012) The Stem Cell Discovery Engine: an integrated repository and analysis system for cancer stem cell comparisons. Nucleic Acids Res 40: D984-D991.

13. Glinsky GV (2008) "Stemness" genomics law governs clinical behavior of human cancer: implications for decision making in disease management. J Clin Oncol 26: 2846-2853.

14. Turner C, Kohandel M (2012) Quantitative approaches to cancer stem cells and epithelial-mesenchymal transition. Semin Cancer Biol 22: 374-378.

15. Turner C, Kohandel M (2010) Investigating the link between epithelialmesenchymal transition and the cancer stem cell phenotype: A mathematical approach. J Theor Biol [Epub ahead of print]

16. Koeva M, Forsberg EC, Stuart JM (2011) Computational integration of homolog and pathway gene module expression reveals general stemness signatures. PLoS One 6: e18968.

17. Wang Z, Liu J, Wang J, Wang Y, Wang N, et al. (2012) Dynamic modeling of genes controlling cancer stem cell proliferation. Front Genet 3: 84

18. Ben-Yehudah A, Easley CA 4th, Hermann BP, Castro C, Simerly C, et al. (2010) Systems biology discoveries using non-human primate pluripotent stem and germ cells: novel gene and genomic imprinting interactions as well as unique expression patterns. Stem Cell Res Ther 1:24.

19. Jiang L, Deberardinis RJ (2012) Cancer metabolism: When more is less. Nature 489: 511-512.

20. Duarte NC, Becker SA, Jamshidi N, Thiele I, Mo ML, et al. (2007) Global reconstruction of the human metabolic network based on genomic and bibliomic data. Proc Natl Acad Sci USA 104: 1777-1782.
21. Tamir Rashid S, Alexander GJ (2012) Induced pluripotent stem cells: from nobel prize to clinical applications. J Hepatol [Epub ahead of print].

22. Saito S, Onuma Y, Ito Y, Tateno H, Toyoda M, et al. (2011) Possible linkages between the inner and outer cellular states of human induced pluripotent stem cells. BMC Syst Biol 1: S17.

23. Jones RJ, Matsui WH, Smith BD (2004) Cancer stem cells: are we missing the target? J Natl Cancer Inst 96: 583-585.

24. Visvader JE, Lindeman GJ (2008) Cancer stem cells in solid tumours: accumulating evidence and unresolved questions. Nat Rev Cancer 8: 755-768.

25. Sehl M, Zhou H, Sinsheimer JS, Lange KL (2011) Extinction models for cancer stem cell therapy. Math Biosci 234: 132-146.

26. Hu Y, Bajorath J (2012) How Promiscuous Are Pharmaceutically Relevant Compounds? A Data-Driven Assessment. AAPS J [Epub ahead of print]

27. Brown D, Superti-Furga G (2003) Rediscovering the sweet spot in drug discovery. Drug Discov Today 8: 1067-1077.

28. Park EJ, Pezzuto JM (2012) Flavonoids In Cancer Prevention. Anticancer Agents Med Chem 12: 836-851

29. Gullett NP, Ruhul Amin AR, Bayraktar S, Pezzuto JM, Shin DM, et al. (2010) Cancer prevention with natural compounds. Semin Oncol 37: 258-281.

30. Guilford JM, Pezzuto JM (2008) Natural products as inhibitors of carcinogenesis. Expert Opin Investig Drugs 17: 1341-1352.

31. Grabley S, Thiericke R (1999) Bioactive agents from natural sources: trends in discovery and application. Adv Biochem Eng Biotechnol 64: 101-154.

32. Pezzuto JM (2011) The phenomenon of resveratrol: redefining the virtues of promiscuity. Ann N Y Acad Sci 1215: 123-130

33. Azmi AS, Mohammad RM, Sarkar FH (2012) Can network pharmacology rescue neutraceutical cancer research? Drug Discov Today 17: 807-809.

34. Sarkar FH (2010) Nutraceuticals and cancer. Preface. Cancer Metastasis Rev 29: 381-382

35. Sarkar FH (2010) Current trends in the chemoprevention of cancer. Pharm Res 27: $945-949$.

36. Sarkar FH, Li Y, Wang Z, Padhye S (2010) Lesson learned from nature for the development of novel anti-cancer agents: implication of isoflavone, curcumin, and their synthetic analogs. Curr Pharm Des 16: 1801-1812.

37. Padhye S, Banerjee S, Chavan D, Pandye S, Swamy KV, et al. (2009) Fluorocurcumins as cyclooxygenase-2 inhibitor: molecular docking, pharmacokinetics and tissue distribution in mice. Pharm Res 26: 2438-2445.

38. Padhye S, Yang H, Jamadar A, Cui QC, Chavan D, et al. (2009) New difluoro Knoevenagel condensates of curcumin, their Schiff bases and copper complexes as proteasome inhibitors and apoptosis inducers in cancer cells. Pharm Res 26: 1874-1880.

39. Dandawate PR, Vyas A, Ahmad A, Banerjee S, Deshpande J, et al. (2012) Inclusion Complex of Novel Curcumin Analogue CDF and $\beta$-Cyclodextrin $(1: 2)$ and Its Enhanced In Vivo Anticancer Activity Against Pancreatic Cancer. Pharm Res 29: 1775-1786

40. Ali S, Ahmad A, Aboukameel A, Bao B, Padhye S, et al. (2012) Increased Ras GTPase activity is regulated by miRNAs that can be attenuated by CDF treatment in pancreatic cancer cells. Cancer Lett 319: 173-181.

41. Bao B, Ali S, Banerjee S, Wang Z, Logna F, et al. (2012) Curcumin analogue CDF inhibits pancreatic tumor growth by switching on suppressor microRNAs and attenuating EZH2 expression. Cancer Res 72: 335-345.

42. Kanwar SS, Yu Y, Nautiyal J, Patel BB, Padhye S et al. (2011) Difluorinatedcurcumin (CDF): a novel curcumin analog is a potent inhibitor of colon cancer stem-like cells. Pharm Res 28: 827-838.

43. Azmi AS, Ali S, Banerjee S, Bao B, Maitah MN, et al. (2011) Network modeling of CDF treated pancreatic cancer cells reveals a novel c-myc-p73 dependent apoptotic mechanism. Am J Transl Res 3: 374-382.

44. Azmi AS, Ahmad A, Banerjee S, Rangnekar VM, Mohammad RM, et al. (2008) Chemoprevention of pancreatic cancer: characterization of Par-4 and its modulation by 3,3' diindolylmethane (DIM). Pharm Res 25: 2117-2124. 
Citation: Muqbil I, Bao GW, El-Kharraj R, Shah M, Mohammad RM, et al. (2012) Systems and Network Pharmacology Approaches to Cancer Stem Cells Research and Therapy. J Stem Cell Res Ther S7:005. doi:10.4172/2157-7633.S7-005

45. Singh-Gupta V, Banerjee S, Yunker CK, Rakowski JT, Joiner MC, et al. (2012) B-DIM impairs radiation-induced survival pathways independently of androgen receptor expression and augments radiation efficacy in prostate cancer. Cancer Lett 318: 86-92.

46. Kong D, Li Y, Wang Z, Banerjee S, Sarkar FH (2007) Inhibition of angiogenesis and invasion by $3,3^{\prime}$-diindolylmethane is mediated by the nuclear factor-kappaB downstream target genes MMP-9 and UPA that regulated bioavailability of vascular endothelial growth factor in prostate cancer. Cancer Res 67: 33103319.

47. Ahmad A, Kong D, Sarkar SH, Wang Z, Banerjee S, et al. (2009) Inactivation of UPA and its receptor UPAR by 3,3'-diindolylmethane (DIM) leads to the inhibition of prostate cancer cell growth and migration. J Cell Biochem 107: 516-527.

48. Ahmad A, Kong D, Wang Z, Sarkar SH, Banerjee S, et al. (2009) Downregulation of UPA and UPAR by 3,3'-diindolylmethane contributes to the inhibition of cell growth and migration of breast cancer cells. J Cell Biochem 108: 916-925.
49. Li Y, Wang Z, Kong D, Murthy S, Dou QP, et al. (2007) Regulation of FOXO3a/ beta-catenin/GSK-3beta signaling by 3,3 '-diindolylmethane contributes to inhibition of cell proliferation and induction of apoptosis in prostate cancer cells. J Biol Chem 282: 21542-21550.

50. Kong D, Banerjee S, Huang W, Li Y, Wang Z, et al. (2008) Mammalian target of rapamycin repression by 3,3 '-diindolylmethane inhibits invasion and angiogenesis in platelet-derived growth factor-D-overexpressing PC3 cells. Cancer Res 68: 1927-1934.

51. Li Y, Wang Z, Kong D, Li R, Sarkar SH, et al. (2008) Regulation of Akt/FOXO3a/ GSK-3beta/AR signaling network by isoflavone in prostate cancer cells. J Biol Chem 283: 27707-27716.

52. Li Y, Kong D, Ahmad A, Bao B, Sarkar FH (2012) Targeting bone remodeling by isoflavone and 3,3'-diindolylmethane in the context of prostate cancer bone metastasis. PLoS One 7: e33011.
This article was originally published in a special issue, Cancer Stem Cells handled by Editor(s). Fazlul Hoque Sarkar, Wayne State University, USA, Asfar Sohail Azmi, Wayne State University, USA 